\title{
Time-dependent corrections to effective rate and event statistics in Michaelis-Menten kinetics
}

\author{
N. A. Sinitsyn $1,2, *$ and Ilya Nemenman $3,+\oplus$ \\ ${ }^{1}$ Theoretical Division, Los Alamos National Laboratory, Los Alamos, NM 87545, USA \\ ${ }^{2}$ New Mexico Consortium, Los Alamos, NM 87545, USA \\ ${ }^{3}$ Department of Physics, Department of Biology, \\ and Computational and Life Sciences Strategic Initiative, \\ Emory University, Atlanta, GA 30322, USA
}

(Dated: July 5, 2021)

\begin{abstract}
We generalize the concept of the geometric phase in stochastic kinetics to a noncyclic evolution. Its application is demonstrated on kinetics of the Michaelis-Menten reaction. It is shown that the nonperiodic geometric phase is responsible for the correction to the MichaelisMenten law when parameters, such as a substrate concentration, are changing with time. We apply these ideas to a model of chemical reactions in a bacterial culture of a growing size, where the geometric correction qualitatively changes the outcome of the reaction kinetics.
\end{abstract}

\section{INTRODUCTION}

Biochemical reactions are typically characterized in stationary in vitro environments with the hope that their measured properties will hold in vivo. There are clearly many important physiological reasons why this extrapolation may fail. In this article, we focus on one particular reason that has little to do with the physiology, but rather derives from the fact that rates of complex chemical reactions may have non-trivial corrections due to slow, adiabatic drift of (internal) kinetic parameters of the system 1 .

The class of phenomena we study is related to the celebrated Berry's phase in driven quantum mechanical systems ${ }^{2}$, which predicted a contribution to the phase of an adiabatically changing wave function in the form of a integral over the parameter trajectory. Since the original Berry's discovery a number of its generalizations were proposed, e.g., to nonabelian and nonadiabatic regimes. Similar geometric phases were also found in other fields, for example, in dissipative dynamics $\underline{\underline{3}-\underline{6}}$.

Recently, new geometric phases where studied in the domain of purely classical stochastic kinetics $^{\underline{7}-12}$. They were shown to be responsible for the stochastic pump and other ratchet-like effects, and thus they are of clear importance for the theory of chemical enzymes, and specifically 
molecular motors operating in strongly stochastic environment $\underline{13,14}$. This finding raises possibilities of various generalizations of the geometric phase. For example, recently its nonadiabatic counterpart was introduced in Ref. $\stackrel{15}{ }$, and it was shown to be responsible for a non-adiabatic current contribution that has no analog under stationary conditions.

In this Letter, we study another generalization of the geometric phase in stochastic kinetics, namely to a nonperiodic evolution in the parameter space. While its quantum and optical versions were explored in a series of studies $\underline{16} \underline{24}$, their role is still largely unclear. In this work, we show that the gauge invariant noncyclic geometric phase in stochastic kinetics can be unambiguously defined, and that it can be naturally interpreted as being responsible for the leading nonadiabatic correction in the expression for stochastic fluxes, which can qualitatively change kinetics of a chemical reaction.

\section{GENERATING FUNCTION FOR THE MICHAELIS-MENTEN REACTION}

The Michaelis-Menten (MM) reaction 25 is the most fundamental and the simplest enzymatic biochemical process. It describes a catalytic conversion of one type of molecules, called the substrate, into another type, called the product, via an intermediate reaction with an enzyme. Schematically the MM reaction can be represented as

$$
S+E \underset{k_{-1}}{\stackrel{k_{1} n_{\mathrm{S}}}{\rightleftarrows}} S E \underset{k_{-2^{n}} \mathrm{p}}{\stackrel{k_{2}}{\rightleftarrows}} E+P
$$

where $S$ and $P$ denote substrate and product respectively, $s$ and $p$ stand for their concentrations, and $E$ is the enzyme molecule. $S$ and $P$ interact via creating a complex $S E$ which is unstable and dissociates either back into $E$ and $S$ or forward into $E$ and $P$. In the simplest version of the MM mechanism, enzymes catalyze the process but are not modified in any reactions. However, generalizations are certainly possible $\underline{26}$.

In their 1913 article 25 , Michaelis and Menten considered a strongly nonequilibrium situation, neglecting the backwards $E+P$ association, which can be done for $n_{\mathrm{p}} \ll k_{1} n_{\mathrm{s}} / k_{-2}$. However, here we keep this reaction for generality. If the number of $S$ and $P$ molecules is much larger than that of the enzymes, the latter have to perform many substrate conversions each in order to change $S$ and $P$ concentrations noticeably. This is traditionally used to simplify the reaction kinetics since one can assert that enzymes operate in a quasi steady state at current substrate and product concentrations.

Stochastic kinetics of the conversion of $S$ into $P$ is conveniently described by the moments generating function $Z(\chi, t)$ (mgf) and the cumulants generating function $S(\chi, t)$ (cgf) defined 
$\operatorname{ass}^{7,27,28}$

$$
Z(\chi, t)=e^{S(\chi, t)}=\sum_{n=-\infty}^{\infty} P_{n} e^{i n \chi},
$$

where $P_{n}$ is the probability to find net $n$ product molecules generated during the observation time $t$ (back conversion is counted with the negative sign). For a small number of enzymes, they can be considered statistically independent over short periods of time, and the cgfs are additive. Thus we will restrict our study only to the case of a single enzyme without loss of generality.

It is convenient to introduce additional generating functions $U_{E}=\sum_{n=-\infty}^{\infty} P_{n E} e^{i n \chi}$ and $U_{S E}=$ $\sum_{n=-\infty}^{\infty} P_{n S E} e^{i n \chi}$, where $P_{n E}$ and $P_{n S E}$ are the probabilities that, at a given time, the net number of generated product molecules is $n$, and the enzyme is in the unbound/bound state. Then the master equation for the entire process is

$$
\begin{aligned}
& \frac{d}{d t} P_{n E}=-\left(k_{1} n_{\mathrm{s}}+k_{-2} n_{\mathrm{p}}\right) P_{n E}+k_{-1} P_{n S E}+k_{2} P_{(n-1) S E}, \\
& \frac{d}{d t} P_{n S E}=-\left(k_{-1}+k_{2}\right) P_{n S E}+k_{1} n_{\mathrm{s}} P_{n E}+k_{-2} n_{\mathrm{p}} P_{(n+1) E} .
\end{aligned}
$$

Multiplying (3) by $e^{i \chi n}$ and summing over $n$ we find the equation for the generating functions:

$$
\frac{d}{d t}\left(\begin{array}{c}
U_{E} \\
U_{S E}
\end{array}\right)=-\hat{H}(\chi, t)\left(\begin{array}{c}
U_{E} \\
U_{S E}
\end{array}\right)
$$

where

$$
\hat{H}(\chi, t)=\left(\begin{array}{cc}
k_{1} n_{\mathrm{s}}+k_{-2} n_{\mathrm{p}} & -k_{-1}-k_{2} e^{i \chi} \\
-k_{1} n_{\mathrm{s}}-k_{-2} n_{\mathrm{p}} e^{-i \chi} & k_{-1}+k_{2}
\end{array}\right) .
$$

If we set $n=0$ at initial moment $t=0$, then the initial conditions for (4) are $U_{E}(t=0)=p_{E}(0)$, and $U_{S E}(t=0)=p_{S E}(0)$, where $p_{E}(0)$ and $p_{S E}(0)$ are probabilities that the enzyme is free/bound, respectively. Additionally, note that $Z(\chi, t)=U_{E}(\chi, t)+U_{S E}(\chi, t)$. Thus the formal solution for the mgf (2) can be expressed as an average of the evolution operator

$$
Z(\chi, t)=\left\langle 1\left|\hat{T}\left(e^{-\int_{0}^{t} \hat{H}(\chi, t) d t}\right)\right| p(0)\right\rangle
$$

where $\langle 1|=(1,1)| p,(0)\rangle=\left(p_{E}(0), p_{S E}(0)\right)^{T}$, and $\hat{T}$ is the time-ordering operator.

Before we proceed with the case where parameters are time dependent, it is instructive to look first at the stationary regime. To simplify (6), one can find normalized left and right eigenvectors $\left\langle u_{0 / 1}|,| u_{0 / 1}\right\rangle$ and corresponding eigenvalues $\epsilon_{0 / 1}$ of the operator $\hat{H}(\chi)$, where indices 0 and 1 correspond to the two eigenvalues with the smallest and the largest real parts, respectively. There is one left and one right eigenvectors for each eigenvalue. 
Every vector, such as $|p(0)\rangle$ can be expressed as a sum of eigenvectors of $\hat{H}(\chi)$, for example,

$$
|p(0)\rangle=\left\langle u_{0} \mid p(0)\right\rangle\left|u_{0}\right\rangle+\left\langle u_{1} \mid p(0)\right\rangle\left|u_{1}\right\rangle
$$

where we define $\langle\alpha \mid \beta\rangle=\alpha_{1} \beta_{1}+\alpha_{2} \beta_{2}$ to be a standard scalar product of two vectors. Substituting (77) into (6) , for the time-independent Hamiltonian we find the steady state mgf,

$$
Z_{\mathrm{st}}(\chi, t)=e^{-\epsilon_{0}(\chi) t+\ln \left(\left\langle 1 \mid u_{0}\right\rangle\left\langle u_{0} \mid p(0)\right\rangle\right)}+e^{-\epsilon_{1}(\chi) t+\ln \left(\left\langle 1 \mid u_{1}\right\rangle\left\langle u_{1} \mid p(0)\right\rangle\right)},
$$

At time scales $t \gg \max \left[1 / k_{-1}, 1 / k_{2}, 1 /\left(k_{1} n_{\mathrm{s}}\right), 1 /\left(k_{-2} n_{\mathrm{p}}\right)\right]$, the second term in (8) is exponentially suppressed in comparison to the first, and the expression for the mgf simplifies to

$$
Z_{\mathrm{st}}(\chi, t) \approx e^{-\epsilon_{0}(\chi) t+\ln \left(\left\langle 1 \mid u_{0}\right\rangle\left\langle u_{0} \mid p(0)\right\rangle\right)}
$$

Terms analogous to $-\epsilon_{0}(\chi) t$ in (9) have been studied previously $\underline{7}$, 28 . The second term is less threaded: this is the boundary term that does not grow with time and depends on the initial conditions and the averaging over the final states of the enzyme. One can disregard it in comparison to the first contribution when $t \rightarrow \infty$. However, we note that its relative effect decays as $1 / t$, i.e., not exponentially. We will keep the boundary term in the following discussion because it will play an important role to restore the gauge invariance of the nonperiodic geometric phase.

At the first look, the boundary term leads to a contradictory result after setting $t \rightarrow 0$, i.e at the initial moment of the evolution. In this limit, the boundary term does not disappear, namely

$$
\left.S_{\text {bnd }}\right|_{t=0}=\ln \left(\left\langle 1 \mid u_{0}(0)\right\rangle\left\langle u_{0}(0) \mid p(0)\right\rangle\right) \neq 0
$$

However, we expect $\left.S_{\mathrm{bnd}}\right|_{t=0}$ to be zero, since $\left.n\right|_{t=0}=0$, so the mgf should be identically equal to unity. The apparent contradiction is resolved by noting that (9) was derived assuming $t \rightarrow \infty$, and it is simply an invalid approximation for $t=0$. In other words, the boundary term is responsible for the initial fast relaxation to the stationary regime. For more insight, one can calculate the contribution of the boundary term to the average number of generated product molecules. Using the normalization condition $p_{S E}(0)=1-p_{E}(0)$ one can find

$$
n_{\mathrm{bnd}}=-\left.i \frac{\left.\partial S_{\mathrm{bnd}}\right|_{t=0}}{\partial \chi}\right|_{\chi=0}=\frac{\left(k_{2}+k_{-2} n_{\mathrm{p}}\right)\left(k_{2}+k_{-1}-K p_{E}(0)\right)}{K^{2}}
$$

where $K=k_{-1}+k_{2}+k_{1} n_{\mathrm{s}}+k_{-2} n_{\mathrm{p}}$. If one assumes that the initial probability $p_{E}(0)$ for the enzyme to be free is at the equilibrium value $p_{E}(0)=\left(k_{2}+k_{-1}\right) / K$, then (11) produces $n_{\text {bnd }}=0$, as expected. To confirm this, one can also derive (11) by a standard master equation approach. 
That is, calculating the average number of new product molecules $n_{\text {bnd }}(t)$, one would find that, after a sufficiently long time,

$$
n(t)=n_{\text {bnd }}+\frac{k_{1} k_{2} n_{\mathrm{s}}-k_{-1} k_{-2} n_{\mathrm{p}}}{K} t .
$$

The second term in Eq. (12) is the average number of the product molecules produced during time $t$ at a steady state. It is the standard prediction of the reversible MM theory, and the first term is a correction, which is nonzero when the initial state of enzymes is not the same as its steady state.

\section{NONCYCLIC GEOMETRIC PHASE IN STOCHASTIC KINETICS}

Assume now that there are several slowly time-dependent parameters in the model. We will group them in a vector $\lambda$. In the case of the MM process, one can view these time-dependent parameters as concentrations of the substrate and the product, $\lambda=\left(n_{\mathrm{s}}, n_{\mathrm{p}}\right)$. However, the discussion in this section is completely general.

Following Ref. $\underline{7}$ we partition the time into small intervals, over which kinetic rates can be considered almost constant. We insert the resolution of the identity operator, $\hat{1}=\left|u_{0}(t)\right\rangle\left\langle u_{0}(t)\right|+$ $\left|u_{1}(t)\right\rangle\left\langle u_{1}(t)\right|$, in (6) after every such an interval. One can find then that the boundary term becomes $S_{\text {bnd }}=\ln \left(\left\langle 1 \mid u_{0}(t)\right\rangle\left\langle u_{0}(0) \mid p(0)\right\rangle\right)$. Importantly, it is no longer gauge invariant, i.e., it is sensitive to the redefinition of eigenstates of the Hamiltonian (5) such as $\left|u_{0}\right\rangle \rightarrow e^{\phi(\lambda)}\left|u_{0}\right\rangle$ and $\left\langle u_{0}\right| \rightarrow\left\langle u_{0}\right| e^{-\phi(\lambda)}$. Therefore, taken alone, it has no direct physical meaning.

It will be convenient to rewrite the boundary term as a sum of a gauge invariant part and a term that is an integral from a pure derivative, i.e.,

$$
S_{\mathrm{bnd}}=\left.S_{\mathrm{bnd}}\right|_{t=0}+\int_{\mathbf{c}} \mathbf{P} \cdot d \lambda, \quad \mathbf{P}=\partial_{\lambda} \ln \left\langle 1 \mid u_{0}\right\rangle,
$$

where $\mathbf{c}$ is the contour in the space of the variable parameters. By analogy with Ref 7 , and including the boundary contribution (13), the mgf in the quasi steady state limit can be written as an exponent of a sum of two terms,

$$
Z(\chi)=e^{S_{\text {geom }}(\chi)+S_{q s t}(\chi)}
$$

where

$$
S_{\mathrm{qst}}(\chi)=-\int_{0}^{t} \epsilon_{0}\left(\chi, t^{\prime}\right) d t^{\prime}+\left.S_{\mathrm{bnd}}\right|_{t=0}
$$

is the quasistationary part of the generating function averaged over time. This is the part that morphs into the steady state result (9) for fixed values of all parameters. 
The other term in (14),

$$
S_{\text {geom }}=\int_{\mathbf{c}}[\mathbf{P}(\lambda)-\mathbf{A}(\lambda)] \cdot d \lambda, \quad \mathbf{A}(\lambda)=\left\langle u_{0} \mid \partial_{\lambda} u_{0}\right\rangle
$$

is the geometric phase contribution responsible for additional reaction events. A is called the Berry connection. $S_{\text {geom }}$ has no analog in the strict steady state regime.

Note that, unlike in Ref. $\underline{\underline{7}}$, we do not assume a periodic evolution of parameters. Therefore, the term involving the integral of the Berry connection $\mathbf{A}$ over the path in the parameter space, $-\int_{\mathbf{c}} \mathbf{A}(\lambda) \cdot d \lambda$, is generally not gauge invariant. However, one can easily check that the non-gaugeinvariant contribution due to the boundary term exactly cancels the non-gauge-invariant part of the contour integral from $\mathbf{A}$.

We further mention that the definition (16) differs somewhat from those used for the non-cyclic geometric phase in quantum mechanics. For example, Refs $\underline{16,17}$ define the noncyclic geometric phase as $\gamma_{\mathrm{gp}}=\int_{\mathbf{c}}[\mathbf{A}(\lambda)-\mathbf{P}(\lambda)] \cdot d \lambda$, where $\mathbf{P}=-\operatorname{Im}\left(\frac{\left\langle u(\lambda(0)) \mid \partial_{\lambda} u(\lambda)\right\rangle}{\langle u(\lambda(0)) \mid u(\lambda)\rangle}\right)$. In the present context, the meaning of such definition is unclear, while the geometric phase defined in (16) is derived directly from the exact representation of the mgf.

Since $\mathbf{P}$ is a pure gauge, it is important only when looking at an evolution along an open path in the parameter space. If the parameter vector $\lambda$ returns to its initial value at the end of the evolution, the expression (16) becomes equivalent to the full-period geometric phase defined in Ref $\underline{\underline{7}}$.

\section{CORRECTIONS TO MICHAELIS-MENTEN LAW}

Consider now the average product creation rate in the MM system under the slow parameter evolution. The average number of new product molecules is $\langle n(t)\rangle=-i(\partial Z(\chi, t) / \partial \chi)_{\chi=0}$. Therefore, just like the full cgf, the average rate of the product production $\langle J\rangle=d\langle n(t)\rangle / d t$ can be written as a sum of the quasistationary $J_{\mathrm{qst}}$ and the geometric $J_{\text {geom }}$ contributions

$$
\langle J\rangle=J_{\text {geom }}+J_{\mathrm{qst}}=\left.\frac{d}{d t} \frac{\partial S_{\text {geom }}}{\partial \chi}\right|_{\chi=0}+\left.\frac{\partial \epsilon_{0}(\chi, t)}{\partial \chi}\right|_{\chi=0} .
$$

The geometric phase is time-dependent only via the time-dependence of the parameter vector $\lambda$. In the case of MM reaction with time-dependent concentrations $n_{\mathrm{s}}$ and $n_{\mathrm{p}}$, the time derivative of the first term in (17) can be expressed as $d / d t \rightarrow\left(d n_{\mathrm{s}} / d t\right) \partial / \partial n_{\mathrm{s}}+\left(d n_{\mathrm{s}} / d t\right) \partial / \partial n_{\mathrm{p}}$. Substituting 
the eigenvectors and eigenvalues of $\hat{H}(\chi, \lambda)$ into (17), we find

$$
\begin{aligned}
J_{\mathrm{qst}} & =\frac{\left(k_{1} n_{\mathrm{s}}(t)\right) k_{2}-\left(k_{-2} n_{\mathrm{p}}(t)\right) k_{-1}}{K}, \\
J_{\text {geom }} & =-\left(k_{2}+k_{-1}\right) \frac{\left(k_{2}+k_{-2} n_{\mathrm{p}}(t)\right)\left(k_{1} \dot{n}_{\mathrm{s}}(t)+k_{-2} \dot{n}_{\mathrm{p}}(t)\right)}{K^{3}} .
\end{aligned}
$$

One can recognize $J_{\text {qst }}$ as the average current for a steady state with fixed values of parameters. In fact, (18) is what is known as the Michaelis-Menten law. However, our results show that this law is not exact when concentrations of the substrate and the product have their own time-dependent evolution. The geometric contribution is the first correction to the Michaelis-Menten kinetics that becomes nonzero when the substrate/product concentrations change with time. Specifically, in the most frequent case $n_{\mathrm{p}} \approx 0$, the average rate of the coarse grained MM reaction per one enzyme becomes

$$
\langle J\rangle \approx \frac{k_{2} n_{\mathrm{s}}}{n_{\mathrm{s}}+\frac{k_{2}+k_{-1}}{k_{1}}}-\left(k_{2}+k_{-1}\right) \frac{k_{2} k_{1} \dot{n}_{\mathrm{s}}(t)}{\left(k_{1} n_{\mathrm{s}}+k_{2}+k_{-1}\right)^{3}} .
$$

That is, even in this case, the time-dependence of the substrate concentration introduces corrections to the reaction rate.

It is possible to understand the result (19) with a simpler approach, which, unfortunately, is hard to generalize for higher current cumulants to demonstrate the geometric nature of the effect for all of them. The probability $p_{E}$ of the enzyme to be unbound evolves according to the master equation

$$
\frac{d}{d t} p_{E}=-\left[k_{1} n_{\mathrm{s}}(t)+k_{-2} n_{\mathrm{p}}(t)\right] p_{E}+\left(k_{2}+k_{-1}\right)\left(1-p_{E}\right),
$$

with the solution

$$
p_{E}(t)=\left(k_{2}+k_{-1}\right) \int_{0}^{t} e^{-\int_{t_{1}}^{t}\left[k_{1} n_{\mathrm{s}}(\tau)+k_{-2} n_{p}(\tau)+k_{2}+k_{-1}\right] d \tau} d t_{1} .
$$

The lower limit in this integral is not important because we work in the adiabatic approximation, which means that the information about the initial state is quickly forgotten. The exponent of the integral over $\tau$ in (22) is then

$$
\begin{aligned}
& e^{-\int_{t_{1}}^{t}\left[k_{1} n_{\mathrm{s}}(\tau)+k_{-2} n_{\mathrm{p}}(\tau)+k_{2}+k_{-1}\right] d \tau} \\
& \quad \approx e^{-\left[k_{1} n_{\mathrm{s}}(t)+k_{-2} n_{\mathrm{p}}(t)+k_{2}+k_{-1}\right]\left(t-t_{1}\right)}\left(1+\frac{k_{1} \dot{n}_{\mathrm{s}}(t)+k_{-2} \dot{n}_{\mathrm{p}}(t)}{2}\left(t-t_{1}\right)^{2}\right) .
\end{aligned}
$$

Performing the remaining integration we find the expression for the probability of the enzyme to be unbound:

$$
p_{E} \approx \frac{k_{2}+k_{-1}}{K}+\frac{\left(k_{2}+k_{-1}\right)\left(k_{1} \dot{n}_{\mathrm{s}}(t)+k_{-2} \dot{n}_{\mathrm{p}}(t)\right)}{K^{3}} .
$$


From (24), one can calculate the average reaction rate and check that indeed, it is the sum of the quasi-stationary and the geometric components determined in (18) and (19),

$$
J(t)=\left(1-p_{E}(t)\right) k_{2}-p_{E}(t) k_{-2} n_{\mathrm{p}}(t)=J_{\mathrm{qst}}+J_{\text {geom }}
$$

\section{GEOMETRIC RATE CORRECTIONS IN A GROWING CELL CULTURE}

The geometric correction (19) is generally much smaller than the main contribution (18) if the number of the enzymes is much smaller than that of the substrates and the products. However, this small correction has very different properties, and can change a system behavior qualitatively under special conditions.

The quasi-steady state contribution to the kinetic rate in (18) can be vanishing due to a symmetry relation, such as the detailed balance condition, which guaranties that all chemical fluxes at the thermodynamic equilibrium state are zero on average. Thus, if a system is slowly driven externally so that it always remains close to the thermodynamic equilibrium, the quasi-steady state approximation will predict zero average product creation. In contrast, the geometric contribution does not have to remain zero, and it will result in a qualitatively novel effect.

To show this, consider the MM reaction with concentrations of substrate and product $n_{\mathrm{s}}$ and $n_{\mathrm{p}}$ large and treated deterministically. Let us suppose that the system is initially in an equilibrium,

$$
k_{1} k_{2} n_{\mathrm{s}}(0)=k_{-1} k_{-2} n_{\mathrm{p}}(0) .
$$

Now suppose that this process happens inside a living cell that grows and divides in its usual cycle. Then the substrate/product molecules in a single cell are diluted by $N(t)$, the number of cells in the descendant colony:

$$
n_{\mathrm{s}}(t)=n_{\mathrm{s}}(0) \frac{N(0)}{N(t)}, \quad n_{\mathrm{p}}(t)=n_{\mathrm{p}}(0) \frac{N(0)}{N(t)}
$$

Since the ratio $n_{\mathrm{s}}(t) / n_{\mathrm{p}}(t)$ is not affected by this time dependent dilution, the system remains near equilibrium, and the quasi-steady state reaction rate remains zero. Thus the average number of new product molecules, produced by a single enzyme is completely determined by the geometric part of the rate (19),

$$
n=\int_{v(0)}^{\infty} d v\left[-\left(k_{2}+k_{-1}\right) \frac{\left(k_{2}+k_{-2} n_{\mathrm{p}} v(0) / v\right)\left(k_{1} \partial_{v}\left(n_{\mathrm{s}}(0) v(0) / v\right)+k_{-2} \partial_{v}\left(n_{\mathrm{p}}(0) v(0) / v\right)\right.}{K^{3}(v)}\right]=
$$


On the one hand, this effect is very small: the average number of new product molecules per one enzyme is a fraction of unity, which compares to a large number of already existing substrate and product molecules. On the other hand, the geometric contribution qualitatively changes the result, predicting on average nonzero amount of new product molecules, which is not expected from the standard MM treatment. If the number of the enzymes in the culture is proportional to the number of cells, and hence grows with time as $N(t)$, this effect will eventually become observable.

The result (28) would be valid only if we could treat concentrations as parameters, changing only due to the external volume growth. In a closed system chemical fluxes eventually should be compensated by the reverse fluxes due to the violation of the steady state condition (26)). Thus the geometric flux should be possible to detect by measuring the deviation of the ratio $n_{\mathrm{s}} / n_{\mathrm{p}}$ from the equilibrium value.

Considering intermediate stages of the culture growth, one can notice that the number of newly produced molecules depends only on the initial and the final cell numbers: that is, the average number of produced proteins depends on the current state of the system, but not on how it got there or where it's going from there. This can be utilized by living organisms in order to control some processes depending on the stage of cell's life cycle. Although this effect is very small, it should be interesting to explore its detectability in vivo and employ it in artificial biochemical circuits design.

\section{DISCUSSION}

In this article, we generalized the notion of the geometric phase in evolution of the mgf to nonperiodic time-dependent processes. For this, the contour integral of the Berry connection had to be supplemented by an extra term restoring the gauge invariance of the geometric contribution to the cgf. This term originates from the boundary contribution responsible for proper description of the initial and final moments of the measurement. For nonequilibrium initial conditions, the boundary terms are responsible for the initial fast relaxation to the enzymatic quasi-steady state. That is, although our approach is adiabatic, it also rigorously captures initial fast relaxation effects.

Our non-periodic geometric phase is different from the ones often encountered in quantum mechanical applications. Its uniqueness follows from the existence of a special gauge that should be imposed in order to describe stochastic kinetics correctly.

We showed that the phase is responsible for nonadiabatic corrections to the standard MichaelisMenten approximation. Such corrections are usually small in comparison to the quasi-steady 
state predictions. However, they explicitly break time-reversal symmetries and, therefore, can produce a qualitatively different result when a chemical system is driven closely to a thermodynamic equilibrium, as in the cell culture growth model that we discussed.

It is unclear whether this effect is of biological relevance. However, we note that we studied only the simplest of its realizations. The introduced non-periodic geometric phase is completely general and should appear practically in any interacting chemical system driven by external fields. Other interesting examples will surely emerge with time. We expect the greatest opportunities for biological relevance in the domain of molecular motors, where geometric effects play an important role as is $\underline{14}$.

It would also be interesting to understand if the nonperiodic geometric phase is related to the existence of fluctuation theorems 29 . Indeed, instead of chemical fluxes, it is possible to use the same formalism to count work or dissipated energy in a driven stochastic system. The absence of anholonomies, such as cyclic geometric phases may indicate the existence of fluctuation relations since then the counting statistics depends only on initial and final values of external parameters, at least in the adiabatic limit. Generalizations of our approach to a nonadiabatic evolution should also be possible since similar generalizations simultaneously to a noncyclic and nonadiabatic evolution in quantum mechanics exist ${ }^{30}$.

\section{Acknowledgments}

N. A. S. was supported by NSF under Grant No. ECCS-0925618 and partially by the US DOE under Contract No. DE-AC52-06NA25396. I. N. was supported by Los Alamos National Laboratory LDRD program during earlier stages of this work. Authors thank Robert Ecke and the entire community of the LANL Center for Nonlinear Studies for creation of a unique collaborative research atmosphere.

* Electronic address: nsinitsyn@lanl.gov

$\dagger$ Electronic address: ilya.nemenman@emory.edu

1 R. Dean Astumian Phys. Chem. Chem. Phys. 11, 9592 (2009).

2 M. Berry Proc. R. Soc. Lond. A 392, 45 (1984).

3 M. L. Kagan, T. B. Kepler and I. R. Epstein, Nature 349, 506 (1991); T. B. Kepler and M. L. Kagan, Phys. Rev. Lett. 66, 847 (1991).

4 N. A. Sinitsyn, and J. Ohkubo, J. Phys. A.: Math. Theor. (2008). 
5 A. S. Landsberg, Phys. Rev. Lett. 69, 865 (1992).

6 N. A. Sinitsyn, V. V. Dobrovitski, S. Urazhdin and A. Saxena, Phys. Rev. B 77, 212405, (2008)

7 N. A. Sinitsyn and I. Nemenman, EPL 77, 58001 (2007).

8 N. A. Sinitsyn and I. Nemenman, Phys. Rev. Lett. 99, 220408 (2007).

9 N. A. Sinitsyn, Phys. Rev. B 76, 1 (2007)

10 J. M. R. Parrondo, Phys. Rev. E 57, 7297-7300 (1998).

11 S. Rahav, J. Horowitz, and C. Jarzynski Phys. Rev. Lett. 101, 140602 (2008).

12 J. Horowitz and C. Jarzynski, J. Stat. Phys. 136917 (2009).

13 Y. Shi and Q. Niu, Europhys. Lett. 59, 324 (2002).

14 D. Astumian, Proc. Natl. Acad. Sci. (USA) 104, 19715 (2007)

15 J. Ohkubo, J. Stat. Mech. P02011 (2008).

16 A. K. Pati, Ann. Phys. 270, 178 (1998).

17 S. R. Jain, A. K. Pati, Phys. Rev. Lett. 80, 650 (1998).

18 E. Sjöqvis Phys. Lett. A 286, 4 (2001).

19 S. Filipp, Y. Hasegawa, R. Loidl, and H. Rauch, Phys. Rev. A 72, 021602(R) (2005).

20 E. Sjqvist, M. Hedstrm, Phys. Rev. A 56, 3417 (1997).

21 X-B. Wang, L. C. Kwek, Y. Liu, C. H. Oh, Phys.Rev. D 63053003 (2001).

22 A. Mostafazadeh, J. Phys. A: Math. Gen. 32, 8157-8171 (1999).

23 S. L. Zhu and Z. D. Wang, Phys. Rev. Lett. 85, 1076 (2000).

24 J. Christian and A. Shimony J. Phys. A: Math. Gen. 26 5551-5567 (1993).

25 L. Michaelis and M. L. Menten, Biochem. Z. 49, 333 (1913).

26 B. P. English et al.,Nat. Chem. Biol. 2, 87 (2006).

27 I. V. Gopich, A. Szabo, J. Chem. Phys. 124, 154712 (2006).

28 D. A. Bagrets and Y. V. Nazarov, Phys. Rev. B 67, 085316 (2003).

29 C. Jarzynsky, Acta Physica Polonica B 29, 1609 (1998).

30 Y. Aharonov and J. Anandan, Phys. Rev. Lett. 58, 1593 (1987). 